\title{
Anomaly in temperature dependence of thermal transport of two hydrogen-bonded glass-forming liquids
}

\author{
A. I. Krivchikov, A. N. Yushchenko, and O. A. Korolyuk \\ B. Verkin Institute for Low Temperature Physics and Engineering of NAN Ukraine, Kharkov 61103, Ukraine \\ F. J. Bermejo and C. Cabrillo* \\ Instituto de Estructura de la Materia, CSIC, and Departamento Electricidad y Electrónica-Unidad Asociada, CSIC, \\ Facultad de Ciencia y Tecnología, Universidad del País Vasco/EHU, P.O. Box 644, E-48080-Bilbao, Spain \\ M. A. González \\ Institut Laue Langevin, BP 156x., F-38042 Grenoble Cedex 9, France
}

(Received 19 March 2007; published 14 June 2007)

\begin{abstract}
The thermal conductivity of two molecular glasses (ethanol and 1-propanol) decrease with increasing temperature up to their glass transitions at $T_{g} \approx 97$ and $98 \mathrm{~K}$, respectively. Within their supercooled liquid phases, the conductivity increases with rising temperature up to a maximum which roughly coincides with the liquidus (or melting temperatures $T_{m} \approx 159 \mathrm{~K}$ and $T_{m} \approx 149 \mathrm{~K}$, respectively). From there on, the conductivity decreases with increasing temperature, a behavior common to most liquids examined so far, exception made of liquid water. The origin of the rather different dependencies with temperature of thermal transport is understood as a competition between phonon-assisted and diffusive transport effects which are amenable to experiments using high resolution quasielastic neutron scattering and visible and ultraviolet Brillouin light-scattering spectroscopies.
\end{abstract}

DOI: 10.1103/PhysRevB.75.214204

PACS number(s): 66.70.+f, 63.50.+x, 65.20.+w, 65.60.+a

\section{INTRODUCTION}

Our current understanding of the mechanisms governing heat transport in disordered matter at low and intermediate $(5-20 \mathrm{~K})$ temperatures relies on phenomenological concepts such as two-level systems (TLSs). Such constructs are able to provide a unified description of all those processes which make such materials poor conductors of heat if compared to their parent crystals, up to temperatures where the thermal conductivity exhibits remarkably mild variations upon further heating and shows the characteristic "plateau." At higher temperatures, a truly quantitative understanding of the effects limiting heat transport has only been achieved for ordered crystals, ${ }^{2}$ whereas equivalent treatments for disordered solids usually rely on generalizations of the TLS model $^{3}$ and usually require explicit account of the interaction of the heat-carrying phonons with other degrees of freedom. ${ }^{4,5}$

In contrast, our knowledge of processes governing heat transport once the solids (i.e., glass and crystal) melt into the supercooled or normal liquid phases remains rather less advanced. This results from the need to take into account contributions of additional processes to those of phonon nature dominant within the solids such as diffusive or crystal nucleation effects. Even within the stable liquid state, the progress in our understanding of this transport property has been modest ${ }^{6}$ which forces all predictions for this industrially important property to rely on empirical correlations ${ }^{7}$ or on results from computer molecular dynamics simulations. ${ }^{6}$ Such a state of affairs contrasts with the wealth of data which mostly concern complicated materials such as polymers, ${ }^{8}$ foodstuffs, ${ }^{9}$ network glasses, ${ }^{10}$ or exotic crystals such as those formed by fullerenes. ${ }^{11}$ In most cases, data analysis is performed in terms of crude Debye models where the temperature dependence of the thermal conductivity is given in terms of the mass density $\rho$, the specific heat $C_{v}$, the average velocity of sound $v$, and the mean free path of the heat carriers $\Lambda$ as ${ }^{12}$

$$
\kappa(T)=\frac{1}{3} \rho C_{v} v \Lambda,
$$

all the three quantities being temperature dependent. Such a result which is derived from kinetic theory, can in purity give a semiquantitative account of experimental data on nearly perfect crystals at relatively low temperatures. Its use, however, has been widespread due to the lack of a more detailed information on the factors limiting heat transport.

For most classical liquids within their stable liquid phases, it is known since the seminal work of Bridgman ${ }^{13}$ that the conductivity decreases with rising temperature, with a few exceptions such as liquid water. Also, the isothermal thermal conductivity rises with density following a nonlinear behavior. Such facts lead Bridgman ${ }^{13}$ to postulate a simple law,

$$
\kappa=2 R v r_{0}^{-2},
$$

given in terms of the gas constant $R$, the sound velocity $v$, and the mean distance between molecular centers of mass $r_{0}$. Such a simple expression fared remarkably well for a whole set of liquid data available at the time. More refined treatments are nowadays common practice which make recourse to computer molecular dynamics simulations. The sought quantities are cast within the Green-Kubo linear response theory which relates the thermal conductivity to an integral taken over the energy-flux vector $\widetilde{J}_{Q}$ so that, 


$$
\kappa(T)=\frac{V}{3 k_{B} T^{2}} \int_{0}^{\infty}\left\langle\widetilde{J}_{Q}(t) \widetilde{J}_{Q}(0)\right\rangle d t,
$$

where $V$ stands for the sample volume and $k_{B}$ is the Boltzmann constant. The explicit form for $\widetilde{J}_{Q}$ depends on the system to be investigated. For a crystal, the correlator shown above can be expressed in terms of sums over phonon mode frequencies and velocities, together with the appropriate relaxation rates. In contrast, for systems such as liquids or crystals composed of a set of $N$ highly mobile particles, the energy-flux vector can be expressed microscopically as a sum of a diffusive (Brownian) term plus a contribution comprising all interactions with the surrounding particles as

$$
V \widetilde{J}_{Q}=\sum_{\mathrm{i}=1}^{N} \widetilde{v}_{\mathrm{i}} e_{i}+\frac{1}{2} \sum_{i=1}^{N} \sum_{j=1}^{N} \widetilde{r}_{i j} \widetilde{F}_{i j} \cdot \widetilde{v}_{j} .
$$

The first term comprises atomic (or molecular) velocities $\widetilde{v}_{i}$ and internal energy $e_{i}$ terms that include kinetic plus internal molecular potential energy contributions. On the other hand, the term including the collective effects is defined in terms of sums running over pairs of particles of products of the instantaneous vectors separating two molecules $\tilde{r}_{i j}$, the total interparticle force $\widetilde{F}_{i j}$, and the molecular velocity $\widetilde{v}_{j}$.

Recently, an explicit evaluation of Eqs. (3) and (4) for liquid ethanol, a system extensively studied both dynamically ${ }^{14}$ and thermodynamically, ${ }^{15}$ has been carried out for temperatures above $T=273 \mathrm{~K}$ by means of computer simulations. ${ }^{16}$ The results which are some $10 \%$ below experiment are able to reproduce the quasilinear decrease with temperature of $\kappa(T)$. The quality of such a prediction is to be compared with the result calculated using Eq. (3) which yields for $T=200 \mathrm{~K}$ a value of $0.166 \mathrm{~W} / \mathrm{K} \mathrm{m}$, which is some $17 \%$ below experiment. Furthermore, such a calculation shows that even at such high temperatures, the contribution from the collective term largely exceeds its diffusive counterpart and, in turn, energy diffusion dominates the latter contribution. Put into different words, the calculation just referred to shows that the decrease of $\kappa(T)$ with increasing temperature is understood as resulting from a significant decrease of the contribution of the collective processes able to propagate heat (i.e., phonons), which is not compensated by diffusive terms which are expected to contribute more as the temperature is increased.

While $\kappa(T)$ is thus becoming amenable to be understood by means of phenomenological or truly microscopic means within the deep glass and normal liquid phases, the physics of heat transport within the temperature (or pressure) region where the normal liquid supercools toward the glassy state is, however, far poorer understood. On the one hand, the extension to temperatures close and below melting of the calculations referred to above is hampered by the rather substantial computer resources involved. On the other hand, experimental data at hand show a rather wide variety of behaviors. Many polymers ${ }^{8}$ show an almost linear increase with temperature of $\kappa(T)$ up to a peak value which roughly corresponds to the glass-transition temperature $T_{g}$, and the same applies to some network glasses. ${ }^{4,5,10}$ Data pertaining some- what to simpler systems such as polyalcohols (glycerol and propylene glycol $)^{17,18}$ show some distinctive features such as a broad minimum at $T_{g}$ and a maximum close to the melting temperature of the parent crystal $T_{m}$. The origin of such features is not entirely clear and has been ascribed to the frequency dependence of the specific heat since their techniques measure the product $C_{p} \kappa$ rather than the thermal conductivity. ${ }^{19}$ To end with, results for the low-density amorphous (LDA) and high-density amorphous (HDA) ices ${ }^{20}$ provide yet another glaring example on the difficulty of understanding heat transport in glassy matter at high temperatures. Data for LDA (Ref. 20) show a decrease with increasing temperature up to about $129 \mathrm{~K}$ following an approximate $\kappa(T) \propto T^{-1 / 2}$ law, suggesting that other mechanisms different from diffuse umklapp scattering are operative here. In stark contrast, $\kappa(T)$ for HDA (Ref. 20) displays a behavior closer to that observed for polymers and polyalcohols, exhibiting a mild increase with increasing temperature up to its stability limit. Curiously enough, transformation of HDA into LDA at about $130 \mathrm{~K}$, which involves a significant decrease in density, leads to a marked increase in thermal conductivity. This contrasts with what one may expect since a state with higher density would yield a higher conductivity. The rather disparate behavior shown by LDA and HDA seems difficult to reconcile here with the view that maintains that the presence of such extrema in $\kappa(T)$ arises from its possible frequency dependence since, as commented on in Ref. 20, the spectra of both amorphous phases are not too dissimilar.

Here, we report on recent results concerning the thermal conductivity of two alcohols, ethyl alcohol $\left(\mathrm{EtOH}, \mathrm{C}_{2} \mathrm{H}_{5} \mathrm{OH}\right)$ and 1-propanol $\left(1 \mathrm{Pr}, 1-\mathrm{C}_{3} \mathrm{H}_{7} \mathrm{OH}\right)$, within their supercooled liquid (SCL) and normal liquid ranges. As it will be made clear below, these materials constitute ideal benchmarks to investigate this elusive thermodynamic quantity because of their relative simplicity compared to polymers, polyalcohols, or amorphous ices. The first material, ethanol ${ }^{14,21}$ has been extensively investigated because the possibility to explore, apart from the well known monoclinic fully ordered crystalline (FOC) modification, three long-lived phases, which are an amorphous solid (or glass), an orientationally disordered crystal (ODC) (or orientational glass) showing static orientational disorder but having translational long-range order (LRO) since the molecules sit at the nodes of a bcc lattice, and a crystal with dynamic orientational disorder [rotatorphase crystal (RPC)] which retains LRO as a bcc lattice where molecular centers of mass sit, still exists. In contrast, no traces of such rich polymorphism are found for $1 \mathrm{Pr}$ and both its structure and dynamics within the glass, crystal, and supercooled liquid phases have been recently studied in detail. ${ }^{21,22}$

\section{EXPERIMENTS}

The measurements were carried out under equilibrium vapor pressure by the steady-state potentiometric method using a special setup..$^{15,23}$ The sample container was a $40 \mathrm{~mm}$ long, $22 \mathrm{~mm}$ diameter stainless steel tube having a copper bottom and a cap. Two $\mathrm{Cu}$ wires with $1 \mathrm{~mm}$ diameter were passed through the container in order to provide good thermal con- 
tact. The preparation of the glass or disordered crystals as carried out following previously described procedures ${ }^{14,22}$ and the melts were prepared either by slow cooling of the liquid or by melting of the glass phases. The SCL was studied either starting from cooling the normal liquid, or by melting the structural glass at the lower end of its temperature range of existence. Typical times required for measurements varied within $5-30 \mathrm{~h}$ per data point, depending on the temperature range, which rules out the influence of any strong time-dependent effects.

A semiquantitative estimation of aging effects on the measured value for $\kappa(T)$ was carried out for the glass and ODC phases of ethanol. The measured values for the thermal conductivity below the respective glass transitions are basically independent of previous thermal history, within experimental error. In other words, the value for this thermodynamic quantity is reproducible within statistics whether the fully disordered glass is prepared by a rapid temperature quench into LN2 or cooling from the normal liquid at a rate of about $6 \mathrm{~K} / \mathrm{min}$. On the other hand, repeated crossings of the $\mathrm{ODC} \rightarrow \mathrm{RPC}$ and a subsequent measurement of $\kappa(T)$ within the glassy phase also yield very close values. The finding is understood as a consequence of the strong phonon-scattering regime achieved at temperatures close to $T_{g}$ which dominates over other plausible phenomena such as those related to physical aging.

Data concerning the stochastic dynamics were already reported. ${ }^{14,22}$ Here, we describe previously unpublished data for the two samples at temperatures across their glass transitions which were measured under conditions of very high resolution in energy transfers using the IN10 and IN16 backscattering instruments at the Institut Laue Langevin, Grenoble, France. The former yielded an energy resolution of about $0.8 \mu \mathrm{eV}$ while the latter delivered a resolution of $1 \mu \mathrm{eV}$ and a range of energy transfers of $\pm 15 \mu \mathrm{eV}$. Sample descriptions, as well as their preparation, were carried out in situ, following procedures already described. ${ }^{14,22}$

\section{RESULTS}

Figure 1 shows the temperature dependencies of the thermal conductivity $\kappa(T)$ for the glass, orientationally and rotationally disordered crystals, and supercooled liquid, as well as normal liquid data for EtOh, as well as data for SCL and normal liquid 1Pr. A glance at Fig. 1 shows that $\kappa(T)$ exhibits a well defined maximum for temperatures close to $T_{m}$ $\approx 159 \mathrm{~K}$ for $\mathrm{EtOH}$ and $T_{m} \approx 149 \mathrm{~K}$ for $1 \mathrm{Pr}$, as well as a broad minimum that matches the calorimetric glass $\rightarrow \mathrm{SCL}$ and ODC $\rightarrow$ RPC transitions ${ }^{14}$ for both materials. The decrease with temperature of $\kappa(T)$ within the glass and ODC phases is expected because of strong scattering of heatcarrying transverse phonons operative at such temperatures ${ }^{15}$ and shows the behavior of these materials similar to LDA. ${ }^{20}$

The presence within the ODC of an underlying crystalline lattice allows us to test the predictive capability of some results where the crystal anharmonicity is explicitly taken into account and yield in the high temperature limit, ${ }^{27}$

$$
\kappa(T)=A \frac{a \Theta_{D}^{2}}{\gamma^{2} T}+B a \Theta_{D},
$$

where $A$ and $B$ are material constants, $a$ is the bcc lattice parameter $(a=5.369 \AA$ for $T=90 \mathrm{~K}), \gamma$ is the Grüneisen con-

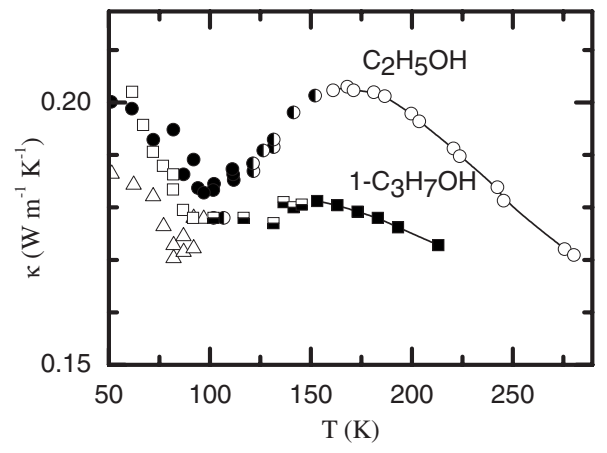

FIG. 1. The thermal conductivity of ethanol across the melting temperature $\left(T_{m}=159 \mathrm{~K}\right)$ and the glass-liquid and ODC-RPC glass transitions $\left(T_{g}=96 \mathrm{~K}\right)$. Filled circles depict data for the orientational (ODC) and rotationally disordered crystals (RPC), open triangles show data for the structural glass, and half-filled circles show data for the supercooled liquid. Open circles with a line depict previous data (Ref. 24). Open squares display data for glassy $1 \mathrm{Pr}$. Data for the supercooled liquid are shown by half-filled squares. Data for the normal liquid range are shown by filled squares with a line.

stant, and $\Theta_{D}$ is the Debye temperature. Data pertaining to the temperature dependence of $a$, as well the value for $\gamma$, have been explicitly measured ${ }^{28,29}$ and the Debye temperature was calculated from low temperature specific heat and yields $\Theta_{D}=229 \mathrm{~K}^{30} \mathrm{~A}$ calculation leaving $A$ and $B$ as adjustable parameters yields a good account of data for the ODC crystal between 50 and $90 \mathrm{~K}$. The parameter values are $A$ $=1.12 \times 10^{6} \mathrm{~W} \mathrm{~m}^{2} \mathrm{~K}^{2}$ and $B=-1.215 \times 10^{6} \mathrm{~W} \mathrm{~m}^{2} \mathrm{~K}^{2}$. Notice that both terms in Eq. (5) contain temperature dependent quantities through the lattice expansion effects. This makes Eq. (5) able to reproduce the $\approx T^{-0.2}$ behavior exhibited by the conductivity since the second term partially compensates the strong $T^{-1}$ term.

The normal liquid ranges depict a quasilinear decrease of the conductivity with increasing temperature, as expected on the grounds of Eq. (2) for decreasing densities and sound velocities. ${ }^{25,26}$ In fact, the data for normal liquid ethanol shows above some $200 \mathrm{~K}$ a linear decrease with temperature with a rate $d \kappa / d T=5.1 \times 10^{-4} \mathrm{~W} / \mathrm{m} \mathrm{K}^{2}$. The result is also to be compared with that calculated from Eq. (3) which yields $d \kappa / d T=1.4 \times 10^{-3} \mathrm{~W} / \mathrm{m} \mathrm{K}^{2}$, mostly due to thermal expansion effects. A milder decrease is also found for 1-Pr within its stable liquid phase. At such temperatures, the decrease in thermal conductivity is understood as resulting from the combined effects of a decrease in density and changes due to the temperature dependence of diffusive plus collective effects (i.e., increasing values for the diffusion coefficients, as well as a concomitant decrease in sound velocity).

The effects of crystal nucleation and growth processes have also been taken into account with quite some care. A previous study on the kinetics of crystallization of SCL ethanol into the bcc rotator-phase crystal by Cabrillo et al. in Ref. 14 has shown that such process reaches a maximum rate at temperatures about $100 \mathrm{~K}$ while crystallization into the stable monoclinic form becomes favorable for temperatures above $127 \mathrm{~K}$. These results also show that, in agreement with estimates based on theories for homogeneous nucle- 


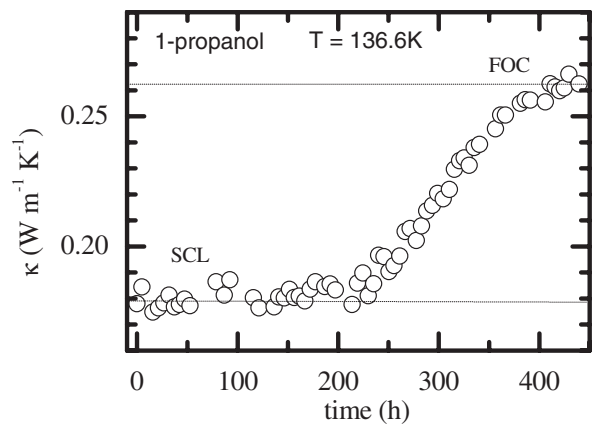

FIG. 2. Time dependence of the measurements of $\kappa(T)$ for 1-propanol within the deeply supercooled liquid state. The increase in conductivity after $200 \mathrm{~h}$ is ascribed to crystal formation as ascertained by $\mathrm{x}$-ray diffraction measurements.

ation, maximal time lags of the order of $10^{2}-10^{3} \mathrm{~s}$ (Ref. 31) are required to begin crystal growth processes and these are far larger than the time taken to perform the experiments. Furthermore, one would expect to find enhanced thermal resistance rather than conductivity due to scattering of acoustic phonons from crystallites, a behavior that goes counter to observation. In fact, detailed results concerning aging effects are shown in Fig. 2, which depicts the time dependence of a measurement on deeply supercooled 1 Pr. Such data show that no significant time dependence is registered for observation times up to some $200 \mathrm{~h}$ which are well beyond those employed for the measurement. The increase in $\kappa(T)$ found after such waiting times are due to onset of crystallization processes as it has been ascertained by diffraction means. At any rate, any significant aging effect to $\kappa(T)$ is expected to be comparable to that explored for glycerol, ${ }^{32}$ where the Brillouin frequency shift was found to increase by some $2 \%$ over a period of 17 days, whereas the quantity having a stronger influence on $\kappa(T)$, that is, the Brillouin linewidth, which provides a measure of phonon-scattering mechanisms, showed no change after an initial rapid decrease. On the other hand, the direct measurement of the relaxation of thermal properties in glasses usually shows that annealing effects are mostly felt by the heat capacity rather than the thermal conductivity. ${ }^{32}$

\section{DISCUSSION}

The strong increase with temperature of $\kappa(T)$ within the SCL for ethanol is understood on the basis of the results for the dynamics measured for the RPC which represents the phase structurally and thermodynamically closest to the SCL. ${ }^{14}$ Choosing the RPC rather than the SCL itself was motivated by the substantially enhanced stability of the former which allowed us to map the dynamics up to temperatures close to its stability limit, $T \approx 127 \mathrm{~K}$. The rotationally disordered crystal exhibits similar trends in $\kappa(T)$ to those found for the SCL and it has also been found to be dynamically close to it by dielectric, light-scattering, and neutron spectroscopies. ${ }^{14,26}$ The upper frame of Fig. 3 shows that crossing the ODC $\rightarrow$ RPC glass transition is accompanied by the onset of relatively fast molecular rotations which were
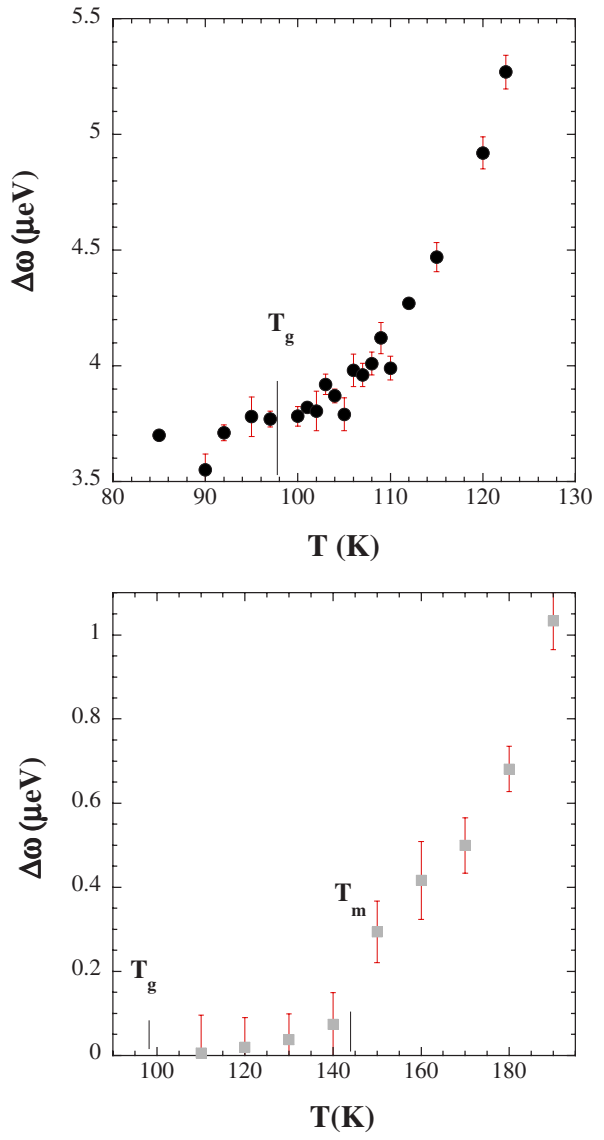

FIG. 3. (Color online) Temperature dependence of the quasielastic neutron scattering linewidths for EtOH (top) as measured on IN16 for the rotator crystal phase and 1-Pr measured using IN10. Data displayed in both figures correspond to an angular average performed to improve signal statistics. The glass and melting transition temperatures for both materials are marked by vertical lines.

characterized in some detail by Jimenez-Ruiz et al. in Ref. 14. Because of their gigahertz frequencies, such molecular motions are expected to contribute significantly to the heat conductivity via the first term of Eq. (4), that is, involving diffusive heat transport. The strong kink in the temperature dependence of the width of the quasielastic spectra $\Delta \omega_{\text {q.el }}(T)$ agrees with the concomitant upward rise in $\kappa(T)$, which is not compensated by the decrease in sound velocity and particle density. Furthermore, as also shown in previous works ${ }^{14}$ the increase in linewidth is accompanied by a corresponding transfer of intensity from the elastic to the quasielastic spectrum, which is interpreted as an increase of the number of particles taking place in rotational motions.

The available data for 1-Pr (Ref. 22) are also shown in Fig. 3. The narrowest component of the quasielastic spectrum reveals the presence of gigahertz motions above $110 \mathrm{~K}$, that is, some $12 \mathrm{~K}$ above $T_{g}$. At these low temperatures, such movements exhibit a noticeable rotational component as commented on in Ref. 22. Such a mixed translationrotational character is retained for all temperatures within the $\mathrm{SCl}$ but dies away within the normal liquid phase. A glance at the data for 1-Pr with respect to that displayed by $\mathrm{EtOH}$ reveals a significant difference. This concerns the far more 
sluggish development of molecular motions within the deeply SCL phase compared to the relatively fast rotations seen in $\mathrm{EtOH}$.

The view just exposed in the above paragraphs also agrees with the absence of noticeable broadenings found for glycerol ${ }^{33}$ below $250 \mathrm{~K}$, that is $1.35 T_{g}$, which corresponds to a region where both $C_{p}$ and $\kappa$ show no anomalous behavior. Notice that in contrast with observations concerning nanosecond time scales, the dynamics of all the three materials across their glass transitions can be followed in detail at time scales involving fractions of a microsecond using Muon spin rotation or by macroscopic means by means of dielectric relaxation. Motions taking place at such larger scales of time seem, however, to contribute little to $\kappa(T)$. Put into real numbers, while dynamic phenomena involving times of the order of a fraction of a microsecond and above take place in the three materials across $T_{g}$, only those within some $1-100 \mathrm{GHz}$ contribute to $\kappa(T)$.

In addition to the diffusive processes just referred to above, $\kappa(T)$ is also sensitive to phonons within the $10-100 \mathrm{GHz}$ range. The lower part of such frequency interval that is expected to contribute most to $\kappa(T)$ can be explored by means of light-scattering spectroscopy, and in fact, detailed studies concerning the behavior with temperature of the hydrodynamic phonons of EtOH (Ref. 26) and glycerol ${ }^{34}$ across their glass transitions have already been published. In both cases, the results show that the phonon frequencies exhibit a kink at roughly $T_{g}$. The temperature dependence of the quantity is more closely related to $\kappa(T)$, that is, the Brillouin linewidth $\Gamma_{B}(T)$, while showing the same qualitative behavior for both materials, that is, displaying maxima at $\approx 150 \mathrm{~K}$ for $\mathrm{EtOH}$ and $\approx 350 \mathrm{~K}$ for glycerol, which are in both cases well above $T_{g}$, but also reveals significant differences. First, such maxima are within the SCL for EtOH but well beyond freezing of the normal liquid for glycerol. Second, all sets of available data reported for EtOH (Refs. 26 and 35) show that $\Gamma_{B}(T)$ strongly increases with increasing temperature well within the deep glass phase, and such an increase becomes more marked for $T>40 \mathrm{~K}$, which lies above the plateau in the thermal conductivity. ${ }^{15}$ Within such region of temperatures, the fractional change of Brillouin frequencies $\Delta \omega_{B} / \omega_{B}$ shows a departure from a $T^{-1}$ law. In contrast, linewidth data for glycerol show a rather mild temperature dependence upon crossing $T_{g}$ and strongly increases above $\approx 250 \mathrm{~K}$, which corresponds to a temperature region where stochastic molecular motions set in. The comparison of both cases points toward the interactions between acoustic modes and stochastic molecular motions as responsible for the observed phenomenology.

The present results also bear some resemblance to observations of amorphous ice and particularly LDA. As shown in Fig. 1, while within the glass or ODC phases of ethanol, as well as in 1-propanol, $\kappa(T)$ decreases with increasing temperature following $\kappa(T) \propto T^{-n}$ with exponents well below $n$ $=-1$, that is, in much the same way as observed for LDA. ${ }^{20}$ The latter value characterizes umklapp scattering in crystals and finding a weaker temperature dependence for the two materials under consideration here, as well as LDA, points toward the operation of additional sources of scattering of heat carriers. In the two cases explored here, molecular motions within the gigahertz range have been found to set in at temperatures comparable or not too far above $T_{g}$. The same phenomenology could not be ascertained for LDA (Ref. 36) because of the crystallization taking place into the crystalline ice phase at the same temperature where the supposed glass transition into a deeply supercooled state has been postulated, that is, $T_{g} \approx 130 \mathrm{~K}$. In contrast, data for HDA resemble those reported for glycerol ${ }^{17,18}$ and other complex materials, ${ }^{8}$ where all sources of variation in $\kappa(T)$ are deemed to arise from those followed by $\rho, C_{v}$, or $v$, which are deemed to be small for the range of temperatures under consideration. In addition and contrary to what was inferred from thermal conductivity measurements, ${ }^{20}$ the spectrum of terahertz excitations of HDA (Ref. 37) depicts a crystal-like response pointing at a high degree of short-range order.

In summary, the present paper reports on direct experimental evidence of rather disparate heat transport mechanisms operative in normal and supercooled liquid phases, which could be related to dynamic processes taking place within gigahertz scales. The results are of particular relevance since measurements of $\kappa(T)$ are shown here to be perhaps the only sensitive tool in investigating the subtle differences in spatiotemporal correlations taking place within a stable liquid and the metastable SCL. Our results thus show common features with that of LDA ice which therefore should no longer be considered as a unique glassy state. ${ }^{20}$

\section{ACKNOWLEDGMENT}

We thank Vadim Manzhelii for fruitful discussions. *jbermejo@we.lc.ehu.es

${ }^{1}$ R. O. Pohl, X. Liu, and E. Thompson, Rev. Mod. Phys. 74, 991 (2002).

${ }^{2}$ E. M. Lifshitz and L. P. Pitaevskii, Physical Kinetics (Pergamon, New York, 1981), chap. 7, p. 292; V. A. Konstantinov, Low Temp. Phys. 29, 422 (2003).

${ }^{3}$ U. Buchenau, Yu. M. Galperin, V. L. Gurevich, D. A. Parshin, M. A. Ramos, and H. R. Schober, Phys. Rev. B 46, 2798 (1992).

${ }^{4}$ F. J. Bermejo, E. Enciso, A. Criado, J. L. Martinez, and M.
Garcia-Hernandez, Phys. Rev. B 49, 8689 (1994).

${ }^{5}$ J. L. Feldman, P. B. Allen, and S. R. Bickham, Phys. Rev. B 59, 3551 (1999).

${ }^{6}$ R. G. Ross, P. Andersson, B. Sundqvist, and G. Backstrom, Rep. Prog. Phys. 47, 1347 (1984); D. M. Heyes and N. H. March, Phys. Chem. Liq. 33, 65 (1996).

${ }^{7}$ D. S. Viswanath and M. B. Rao, J. Phys. D 3, 1444 (1970); C. M. Rodenbusch, D. S. Viswanath, and F. Hsieh, Ind. Eng. Chem. Res. 38, 4513 (1999). For liquid metals, see C. Y. Ho, R. W. 
Powell, and P. E. Liley, Thermal Conductivity of Elements: A Comprehensive Review (American Chemical Society, Washington, DC/American Institute of Physics, New York, 1974), Vol. 3, Suppl. 1, p. 1.

${ }^{8}$ K. Eiermann and K.-H. Hellwege, J. Polym. Sci. 57, 99 (1962); B. Sundqvist, O. Sandberg, and G. Bäckström, J. Phys. D 10, 1397 (1977); T. Bhowmick and S. Pattanayak, Cryogenics 30, 116 (1990); P. Dashora, Phys. Scr. 49, 611 (1994); O. Sandberg and G. Bäckström, J. Polym. Sci., Part B: Polym. Phys. 18, 2123 (1994); C. L. Choy, K. W. Kwok, W. P. Leung, and F. P. Lau, ibid. 32, 1389 (2003); K. Venkateshan and G. P. Johari, J. Chem. Phys. 125, 054907 (2006).

${ }^{9}$ W. Yang, T. J. Siebenmorgen, T. P. H. Thielen, and A. G. Cnossen, Biosys. Eng. 84, 193 (2003).

${ }^{10}$ I. I. Buridyan, E. A. Sonokosov, L. D. Tsirulik, and I. S. Feschenko, Inorg. Mater. 37, 773 (2001).

${ }^{11}$ R. C. Yu, N. Tea, M. B. Salamon, D. Lorents, and R. Malhotra, Phys. Rev. Lett. 68, 2050 (1992); O. Andersson, A. Soldatov, and B. Sundqvist, Phys. Lett. A 206, 260 (1995).

${ }^{12}$ G. P. Srivastava, The Physics of Phonons (Hilger, Bristol, 1990), p. 135.

${ }^{13}$ P. W. Bridgman, Proc. Natl. Acad. Sci. U.S.A. 9, 341 (1923). For a recent appraisal, see A. R. Regel, I. A. Smirnov, and E. V. Shadrichev, Phys. Status Solidi A 5, 13 (2006).

${ }^{14}$ M. A. Ramos, S. Vieira, F. J. Bermejo, J. Dawidowski, H. E. Fischer, H. Schober, M. A. Gonzalez, C. K. Loong, and D. L. Price, Phys. Rev. Lett. 78, 82 (1997); M. Jimenez-Ruiz, A. Criado, F. J. Bermejo, G. J. Cuello, F. R. Trouw, R. FernandezPerea, H. Löwen, C. Cabrillo, and H. E. Fischer, ibid. 83, 2757 (1999); C. Cabrillo, F. J. Bermejo, M. Jimenez-Ruiz, M. T. Fernandez-Diaz, M. A. Gonzalez, and D. Martin y Marero, Phys. Rev. B 64, 064206 (2001); F. J. Bermejo, C. Cabrillo, M. A. Gonzalez, and M. L. Saboungi, J. Low Temp. Phys. 139, 567 (2005).

${ }^{15}$ A. I. Krivchikov, A. N. Yushchenko, V. G. Manzhelii, O. A. Korolyuk, F. J. Bermejo, R. Fernandez-Perea, C. Cabrillo, and M. A. Gonzalez Phys. Rev. B 74, 060201(R) (2006).

${ }^{16}$ J. Petravic, J. Chem. Phys. 123, 174503 (2005).

${ }^{17}$ N. O. Birge, Phys. Rev. B 34, 1631 (1986).

${ }^{18}$ D. G. Cahill and R. O. Pohl, Phys. Rev. B 35, 4067 (1987).

${ }^{19}$ O. Andersson, Int. J. Thermophys. 18, 195 (1997).

${ }^{20}$ O. Andersson and H. Suga, Phys. Rev. B 65, 140201(R) (2002); O. Andersson and A. Inaba, J. Chem. Phys. 122, 124710 (2005); , Phys. Chem. Chem. Phys. 7, 1441 (2005).

${ }^{21}$ C. Cabrillo, F. J. Bermejo, and S. F. J. Cox, Phys. Rev. B 67, 184201 (2003); F. J. Bermejo, I. Bustinduy, M. A. Gonzalez, S. H. Chong, C. Cabrillo, and S. F. J. Cox, ibid. 70, 214202 (2004).

${ }^{22}$ Data concerning the dynamics as studied by dielectric relaxation and quasielastic neutron scattering are given in F. J. Bermejo, W. S. Howells, M. Jimenez-Ruiz, M. A. Gonzalez, D. L. Price, M. L. Saboungi, and C. Cabrillo, Phys. Rev. B 69, 174201 (2004).
The frequency spectra for glass and crystal are given in C. Talon, G. J. Cuello, M. A. Gonzalez, F. J. Bermejo, C. Cabrillo, and R. Connatser, Chem. Phys. 292, 263 (2003). For the structures for the glass and crystal phases see C. Talon, et al., Phys. Rev. Lett. 88, 115506 (2002).

${ }^{23}$ A. I. Krivchikov, B. Y. Gorodilov, O. A. Korolyuk, V. G. Manzhelii, H. Conrad, and W. Press, J. Low Temp. Phys. 139, 693 (2005); A. I. Krivchikov, V. G. Manzhelii, O. A. Korolyuk, B. Y. Gorodilov, and O. O. Romantsova, Phys. Chem. Chem. Phys. 7, 728 (2005).

${ }^{24}$ G. H. Muhamedzianov and A. G. Usmanov, Teploprovodnost Organicheskih Zhydkostej (Thermal Conductivity of Organic Liquids) (Khimiya, Leningrad, 1971), p. 116; N. B. Vargaftik, Handbook of the Physical Properties of Liquids and Gasses, 2nd ed. (Springer-Verlag, Berlin, 1983).

${ }^{25}$ T. F. Sun, J. A. Schouten, N. J. Trappeniers, and S. N. Biswas, J. Chem. Thermodyn. 20, 1089 (1988); O. Haida, H. Suga, and S. Seki, ibid. 9, 1133 (1977).

${ }^{26}$ A. Srinivasan, F. J. Bermejo, and A. de Bernabé, Mol. Phys. 87, 1439 (1996); J.-H. Koh and S. Kojima, J. Non-Cryst. Solids 307-310, 154 (2002); Y. Takagi, Physica B 263-264, 306 (1999).

${ }^{27}$ D. N. Sahu and P. K. Sharma, Phys. Rev. B 28, 3200 (1983); V. A. Konstantinov, V. G. Manzhelii, S. A. Smirnov, and A. M. Tolkachev, Sov. J. Low Temp. Phys. 14, 104 (1988).

${ }^{28}$ M. A. Gonzalez, E. Enciso, F. J. Bermejo, and M. Bée, Phys. Rev. B 61, 6654 (2000).

${ }^{29}$ H. E. Fischer, F. J. Bermejo, G. J. Cuello, M. T. Fernandez-Diaz, J. Dawidowski, M. A. Gonzalez, H. Schober, and M. JimenezRuiz, Phys. Rev. Lett. 82, 1193 (1999).

${ }^{30}$ C. Talon, et al., Phys. Rev. B 58, 745 (1998).

${ }^{31}$ I. Gutzow and J. Schmelzer, The Vitreous State, Thermodynamics, Structure, Rheology and Crystallization (Springer, Berlin, 1995), Chapt. 6, p. 269.

${ }^{32}$ R. S. Miller and R. A. MacPhail, J. Chem. Phys. 106, 3393 (1997). An assessment of the strength of annealing effects on thermal diffusivity of phosphate glasses is given in Y. Hiki, H. Takahashi, and Y. Kogure, Physica B 263-264, 353 (1999) and shows that the observed effects arise from changes in the specific heat rather than from changes in thermal conductivity.

${ }^{33}$ G. J. Cuello, F. J. Bermejo, R. Fayos, R. Fernandez-Perea, F. Trouw, C. Tam, and H. Schober, Mol. Phys. 93, 341 (1998).

${ }^{34}$ W. T. Grubbs and R. A. MacPhail, J. Chem. Phys. 100, 2561 (1994); A. Giugni and A. Cunsolo, J. Phys.: Condens. Matter 18, 889 (2006).

${ }^{35}$ A. Criado, M. Jimenez-Ruiz, C. Cabrillo, F. J. Bermejo, M. Grimsditch, H. E. Fischer, S. M. Bennington, and R. S. Eccleston, Phys. Rev. B 61, 8778 (2000).

${ }^{36}$ M. M. Koza, B. Geil, H. Schober, and F. Natali, Phys. Chem. Chem. Phys. 7, 1423 (2005).

${ }^{37}$ M. M. Koza, H. Schober, B. Geil, M. Lorenzen, and H. Requardt, Phys. Rev. B 69, 024204 (2004). 